\title{
Fungal Keratitis: Update for 2014
}

\author{
Donald Stone $\cdot$ Jeremy F. Tan
}

Published online: 22 July 2014

(C) Springer Science + Business Media New York 2014

\begin{abstract}
Fungal keratitis is a challenging problem worldwide, and many obstacles remain to the effective prevention, diagnosis, and therapy of this vision-threatening condition. Recent research has highlighted progress in each of these facets of the clinical approach to keratomycosis. Increased awareness of contact lens-associated fungal keratitis has prompted regulators to pay greater attention to testing of contact lens solutions under realworld conditions. Related research has also delved into the mechanisms of fungal biofilm formation and related therapeutic targets. Advances in polymerase chain reaction methodologies promise to increase the rapidity and accuracy of diagnosis, thereby decreasing the delay in instituting appropriate therapy. Antifungal pharmacologic agents remain the first line of therapy, with the newer azole antifungal voriconazole being utilized in innovative ways, such as via intrastromal injection. Large prospective studies such as the Mycotic Ulcer Treatment Trial have helped delineate optimal treatment algorithms, and variations in surgical techniques and postoperative regimens continue to be refined. Natamycin may still be more effective than other recently utilized antifungals for the treatment of Fusarium infections, but treatment failure is still a concern. Post-keratoplasty immunosuppression may benefit from the use of topical calcineurin inhibitors such as cyclosporine and tacrolimus, given their intrinsic antifungal properties. It can be argued that the prevention, diagnosis, and treatment of fungal keratitis lag behind the state of the art for bacterial keratitis, but dramatic improvements are undoubtedly on the horizon.
\end{abstract}

D. Stone $(\bowtie) \cdot$ J. F. Tan

Department of Ophthalmology, Dean McGee Eye Institute, University of Oklahoma, Oklahoma City, OK, USA

e-mail: Donald-Stone@dmei.org
Keywords Fungal $\cdot$ Keratitis - Keratomycosis . Antifungal · Natamycin · Voriconazole $\cdot$ PCR · Cornea

\section{Introduction}

Fungal keratitis (FK), or keratomycosis, is an infectious process of the cornea which is a significant cause of visual impairment and utilization of health care resources in the industrialized world as well as in developing countries. It often represents a diagnostic and therapeutic challenge, leading to delay in initiation of appropriate anti-fungal treatment; failure of medical therapy is not uncommon, requiring therapeutic keratoplasty in some cases. A recent study shows that cornea specialists were only able to differentiate between bacterial and fungal etiologic agents based on visual appearance $66 \%$ of the time $(p<0.001)[1]$, and the appropriate medical and surgical therapy continues to be the subject of research and debate.

Fungal keratitis also proves to be an economic burden on society. A recent Thai study segregated the cost of treating different forms of infectious keratitis by expense of drug, surgery, service, laboratory, and room with FK accruing the greatest expense by far $(p \leq 0.001)$. A similar relative expense has been found in studies from South India and Australia [2-4]. These studies, which highlight the economic effects of fungal mycoses, do not include the economic effects of visual disability, likely underestimating the true costs of this condition.

\section{Epidemiology}

There are numerous studies and case reports that have described unusual or novel species of fungi associated with 
FK. A 2012 article provides a good summation, finding 144 species of fungi from 92 genera as a cause of keratitis. These numbers are compared to the much fewer 42 different genera of bacteria, 4 genera of protozoa, and 4 types of viruses that were documented as causative agents of infectious keratitis. Still, the causative agent in the majority of FK cases comes from only a few genera; namely Fusarium, Aspergillus, and Candida [5]. In Bangkok Thailand, Shandong China, and South India, Fusarium has been reported to be the most prevalent [6-8]. In the United States Fusarium, Aspergillus, and Candida have been reported as the most common agents in Miami, Minneapolis and Philadelphia, respectively [9].

Re-classification: A "New” Fungus

Microsporidia have been described as a protozoan capable of causing keratoconjunctivitis; however, recent evidence suggests that it should be reclassified as a fungus [10]. Though classically found in immunocompromised individuals, it can be pathogenic in immunocompetent patients as well [11•].

The organism can penetrate into the cornea resulting in a stromal keratitis. A recent summation documents an indolent course, with a mean duration of 332 days from initial onset of symptoms. Often proving to be a diagnostic challenge, $55 \%$ of the compiled patients were initially diagnosed with herpes simplex stromal keratitis. Trauma was reported as the most common predisposing factor, but no specific event was identified in over half. The definitive diagnosis came from microscopic examination of corneal scrapings with $10 \%$ $\mathrm{KOH}$ or calcofluor white under fluorescent light. There was no defined successful medical treatment, though a combination of topical antiparasitic agents such as polyhexamethylene guanidine (PHMB) and chlorhexidine with or without oral albendazole or itraconazole was attempted. Anti-retroviral therapy is suggested in those infected with HIV, and one patient in whom a lamellar keratoplasty was attempted developed a recurrence and subsequent need for penetrating keratoplasty $[10,11 \cdot 12]$.

\section{New Highlights in Pathophysiology}

\section{Host Factors}

\section{Cytokines and Innate Immunity}

There is an intricate cytokine signaling cascade ultimately leading to activation of Dectin-1 and toll-like receptor 4 (TLR4) in neutrophils that appears to be key to the host response, and therefore the severe corneal inflammatory response that leads to opacification [13]. A study among a Han Chinese population of patients with FK compared controls found a particular TLR4 allele that was associated with an increased risk of developing FK [14].

\section{Contact Lens-Associated Fungal Keratitis}

In the mid 2000s, there was an outbreak of Fusariumrelated cases of FK associated with ReNu with MoistureLoc, which has subsequently been removed from the US market [15]. Recent studies point toward the storage of the anti-microbial agent alexidine in its plastic containers at higher than room temperatures $\left(42\right.$ vs. $\left.56^{\circ} \mathrm{C}\right)$ as the reason for decreased effectiveness [16]. A recent comment from the same author further states that this temperature difference in the plastic containers led to decreased concentration of the agent in solution (2.8 times less) and a corresponding higher concentration in the walls of its plastic containers (3.1 times higher) [17••].

Due to this event, there has been further study into the fungicidal activity of cleaning and storage solutions. One study reveals that the lens type and its soaking time significantly influences the fungicidal activity of PHMB in multi-purpose cleaning solutions. There is an increased focus on appropriate testing of the efficacy of contact lens cleaning solutions, specifically related to preventing fungal contamination [18].

As one would expect, the behavior of lens wearers themselves confounds the efficacy of cleaning solutions. In a recent study, only 11 out of 52 patients were fully compliant with uncontaminated lens cases. Male gender, longer lens case use and swimming in a pool while wearing lenses were positive predictors for contamination, while regular lens solution replacement was a negative predictor [19].

Fungal Physiology and Pathogenicity

In a recent study on fungal biofilm formation, $F$. solani formed a biofilm in vitro by $24 \mathrm{~h}$ while other species formed at $48 \mathrm{~h}$. There was a clear increase in the minimum inhibitory concentration (MIC) of all antifungal agents tested (amphotericin B, voriconazole, itraconazole, fluconazole, terbinafine, and natamycin) for all three studied fungi as their biofilms developed; specifically for fusarium the MIC doubled at $24 \mathrm{~h}$ and again at 48 [20•]. This highlights a possible mechanism of pathogenesis of infection, but also suggests that an ability to disrupt the biofilm may prove useful in increasing antibiotic efficacy.

\section{Update on Diagnostic Tools}

A cohort of cornea specialists from the United States and India were asked to differentiate between fungal and bacterial keratitis. As a group, they were only able to 
Table 1 A comparison of reported and extrapolated sensitivities and some specificities of diagnostic methods for fungal keratitis, from recent literature

\begin{tabular}{llll}
\hline Diagnostic method & $\begin{array}{l}\text { Sensitivity } \\
(\%)\end{array}$ & $\begin{array}{l}\text { Specificity } \\
(\%)\end{array}$ & $p$ value \\
\hline Smears & $65.91^{\mathrm{d}} 20.0^{\mathrm{e}}$ & & \\
$10 \% \mathrm{KOH}$ & $44.83^{\mathrm{a}}$ & 17.95 & $<0.05$ \\
Methylthioninium & $70.69^{\mathrm{a}}$ & 34.61 & $<0.05$ \\
chloride & & & \\
Culture & $59.09^{\mathrm{d}}, 40.0^{\mathrm{e}}$ & & \\
Solid culture media & $98.38^{\mathrm{c}}$ & & \\
Blood agar & $78.9^{\mathrm{b}}, 92.72^{\mathrm{c}}$ & & \\
Chocolate agar & $52.6^{\mathrm{b}}, 84.74^{\mathrm{c}}$ & & \\
Sabourad dextrose agar & $100^{\mathrm{b}}, 79.66^{\mathrm{c}}$ & & \\
Liquid media & $83.87^{\mathrm{c}}$ & & \\
Brain heart infusion & $72.41^{\mathrm{c}}$ & & \\
Thioglycolate & $32.65^{\mathrm{c}}$ & & \\
Robertson's cooked & $55.55^{\mathrm{c}}$ & & \\
meat & & & \\
PCR & $90.91^{\mathrm{d}}, 93.3^{\mathrm{e}}$ & & \\
PCR HRM & $100^{\mathrm{a}}$ & 100 & \\
Dot hybridization assay & $100^{\mathrm{a}}$ & 96.7 & \\
\hline
\end{tabular}

a See Refs. [21], [31], [33]

b See Ref. [22]

c See Ref. [72]

d See Ref. [26]

e See Ref. [25]

distinguish between bacterial and fungal etiologies $66 \%$ of the time, and in the 39 fungal cases, the clinicians predicted genus in only $27 \%$ of cases and species in an even fewer $8 \%$. The data from this study in conjunction with the aggressive and recalcitrant nature of fungal infections highlight the need for accurate and rapid diagnostic tests. Table 1 summarizes the reported specificity and sensitivity of the diagnostic modalities described recently.

\section{Stains}

Methylthioninium chloride (MC) was compared to a $10 \%$ $\mathrm{KOH}$ smear in detecting culture-positive fungal elements. In this study, MC displayed superiority of sensitivity at $70.69 \%$ and specificity at $34.61 \%$ [21].

\section{Culture}

A recently published article affirms the benefit of utilizing various media when trying to isolate the organism in suspected FK. Though recent reports alluded to the ability of blood agar (BA) as a universal media, FK grew only $78.9 \%$ of the time on BA, requiring Sabourad's agar (SBA) to reach $100 \%$ sensitivity for culture-positive organisms (comparison in Table 1) [22].
There has also been a recent evaluation of solid and liquid culture media. A comparison of brain heart infusion broth, thioglycolate broth, and Robertson's cooked meat broth was performed, comparing them to three solid media (BA, SBA, and chocolate). In this cohort, 61 out of 62 cases of culture-positive FK were diagnosed with solid media alone. However, upon determining which of the solid media types yielded the most sensitive results, the yield of BA was higher than SBA. These articles suggest a complementary role of the various culture media, with variation in results presumably based on the organisms and protocols being utilized.

\section{Confocal Microscopy}

Confocal microscopy is a rapid, noninvasive in vivo testing modality. A recent case series of 6 patients with positive fungal smear or culture were evaluated using the Heidelberg Retina Tomograph II-Rostock Cornea Module (HRT II-RCM). They were able to detect the fungal agent in all 6 cases and monitor the morphological change as treatment progressed [23]. A more recent review article does point out drawbacks of the current technology; two specific limitations include expense of the technology, as well as a small field of view for examination: a $400 \times 400$ um field for the aforementioned scanner [24].

\section{Polymerase Chain Reaction (PCR)}

PCR has been compared to the conventional diagnostic modalities of smear and culture in recent studies, displaying greater sensitivity (comparison in Table 1) and rapid results [25, 26].

Previous PCR techniques were unable to distinguish between filamentous and yeast species and required complex steps for amplification [27-29]. Real-time Taqman PCR is able to better characterize the fungal species, but is expensive, due in part to the requirement for a unique probe to search for a single fungal type per reaction [30].

The High Resolution Melting (HRM) PCR analysis technique utilizes only two different forward primers, CandUn and FilamUn, with one reverse primer FungUn. This technique tested negative for all the samples that were suspected of having an infectious agent other that fungi, detected all the culture-positive fungi, and also discovered 7 out of 10 suspected fungal but culture-negative samples, only 4 of which were detected by direct microscopic examination. This technique was able to detect and differentiate between yeast and filamentous fungi at 0.1 colony forming units/ul and in less than $2.30 \mathrm{~h}$ after DNA extraction from the sample. HRM PCR with these primers is able to distinguish filamentous from yeast fungi, as well as between 7 different strains of yeast [31]. 
In the dot hybridization assay, the process as reported involves obtaining a corneal scraping sample, isolating the specimen DNA, amplifying the product using PCR techniques, then visually inspecting the reaction between the product and predefined substrates [32]. In comparison to culture, the technique displayed greater sensitivity (100 vs. $50 \%$ respectively). Specificity was $96.7 \%$ with the dot hybridization assay versus $100 \%$ with routine culture as the gold standard in a small study [33].

\section{Clinical Outcomes}

More advanced age, worse visual acuity at presentation, larger infiltrate, and pigmentation of the ulcer are statistically significant variables that were associated with poorer final visual acuity in FK. Time to re-epithelialization was extended with a larger initial infiltrate, old age, and female gender [34]. Our group observed delayed time to resolution in patients who reported smoking tobacco [35]. Of particular importance, larger infiltrate size significantly increases the risk to perforation [36].

In a comparison of bacterial and FKs, data revealed a larger residual infiltrate size from fungal causes at 3 months, a slower time to re-epithelialize (median difference $\sim 7.5$ days), and a greater risk of perforation in FK. Also, there was a trend for worse visual acuity from fungal etiologic causes, but no statistically significant difference [37].

It has also been found that corneal sensation may be decreased after fungal infection, supported by in vivo confocal microscopy showing decreased corneal nerve length as well as nerve counts [38].

A relatively common clinical concern, but uncommon occurance, is the progression from keratitis from any etiologic agent to endophthalmitis. In a large study with over 9,900 eyes with keratitis, only 49 progressed to cultureproven endophthalmitis $(0.5 \%)$. However, fungus was the most common agent in the endophthalmitis cases. Risk factors to progression were topical steroid use (76\%), previous surgery (61\%), corneal perforation (35\%), dry eye $(31 \%)$, relative immune compromise $(20 \%)$, organic matter trauma (18\%), and contact lens wear (6\%). Once this progression occurs, outcomes were poor with $31 \%$ resulting in enucleation or evisceration [39].

\section{Therapeutic Alternatives}

\section{Topical Anti-fungal Therapy}

With the knowledge that the organisms responsible for infectious keratitis are diverse and unpredictably sensitive to the available antifungal agents, there is an apparent need for data to guide therapeutic options in any individual as well as on a societal scale. Several studies have addressed the question of voriconazole as an emerging drug of choice [40, 41]. A head to head comparison was analyzed in the MUTT study, randomizing 323 patients with smear-positive filamentous fungal ulcers to treatment with topical $1 \%$ voriconazole (VCN) v. $5 \%$ natamycin (NMN) hourly [42••].

The first outcome measure addressed was best spectacle corrected visual acuity (BSCVA). Despite expert belief that there would be no difference between treatment with VCN or NMN with even a slight skew toward favoring VCN [43], NMN treated eyes read 1.1 lines better than VCN at 3 weeks and 1.8 lines better at 3 months. Further subgroup analysis of Fusarium v. non-fusarium causes revealed that in fusarium cases, eyes treated with NMN saw 4.1 lines better than VCN-treated eyes $(p<0.001)$. There was no difference in BSCVA between the two treatment arms in the non-fusarium cases. A similar pattern of NMN-treated eyes fairing better in terms of residual scar size is noted, again seemingly due to fusarium cases. As for the need for therapeutic penetrating keratoplasty (TPK), it was again the fusarium cases that showed a decreased odds ratio to perforation and need of TPK when treated with NMN.

The fungal isolates from MUTT were then tested in vitro; in comparison to other filmentous species, fusarium reveals higher MICs to voriconazole [44•]. However, the population that was studied were from a region in Southern India with susceptibility patterns that may not extrapolate to other patient populations. Data on MIC may be important, as another study also reported that higher MIC to natamycin was associated with an increased risk of perforation [45]. At this time, the extended time to obtain sensitivity assays remains a barrier to clinical utilization.

\section{New Topical Anti-fungal Preparations}

In some developing countries, the typical anti-fungals are unavailable due to cost and instability. Topical Clotrimazole- $\beta$-cyclodextrin (CBC) was compared to amphotericin $\mathrm{B}$ (ampB). There was on average 1 week shorter duration of treatment in the CBC-treated eyes. The authors also reported greater efficacy in cases due to Candida sp., while there was no significant difference when a filamentous fungus was the causative agent. However, it is noted that this study was performed in comparison to retrospective data in the ampB arm [46].

A novel liposomal preparation of fluconazole for topical application demonstrated efficacy in rabbit models [47] and a recent study of 11 patients with proven Candida keratitis [48]. Some level of improvement was noted, but with the small study size and lack of control or comparison arms it is difficult to gauge its place in the therapeutic armamentarium. 
Intrastromal/Intracameral Anti-fungal Treatment

Multiple case reports and small series have examined the role of intrastromal delivery of antifungal therapies. A rabbit model was used to evaluate the pharmacodynamics and toxicity of intrastromal injection of ampB. The mean concentrations found in the cornea after $10 \mathrm{ug}$ were injected showed a peak at $129.96 \mathrm{mg} / \mathrm{g}$ at $30 \mathrm{~min}$, then steadily declining to a level of $52.74 \mathrm{mg} / \mathrm{g}$ after 7 days. However, aqueous humor drug concentration was much lower initially and displayed a much more precipitous drop after only 1 day from 253.3 to $30.2 \mathrm{ng} / \mathrm{ml}$. Corneal toxicities from the drug itself did not appear until the drug dosage was increased to $20 \mathrm{ug}$ [49].

The use of intrastromal voriconazole was reported in a series of patients $(n=3)$ who were not responding to topical therapies [50]. However, in a separate study of persistent cases of FK that were not responding to NMN, $\mathrm{VCN}$ was added to the regimen in either the topical or intrastromal form, and healing times revealed no statistical difference [51].

In another small series $(n=5)$, a combination of intracameral and topical VCN was utilized in eyes presenting with FK with endothelial exudates. The authors proceeded with intracameral $\mathrm{VCN}$ as the loading dose and topical $\mathrm{VCN}$ as the maintenance dose, as the pharmacodynamics of intracameral VCN demonstrate a half-life of only $22 \mathrm{~min}$ after injection [52]. The authors attained resolution of infection with a mean healing time of 5.8 weeks in all 5 eyes without recurrence at 3 months. However, residual scarring dependent upon central corneal involvement left highly variable BSCVA from 20/40 to count fingers. Though the authors allude to use of intracameral amphotericin B in the literature as causing side effects of anterior chamber inflammation, pain, and cataracts, their use of VCN for injection demonstrated no adverse effects [53].

\section{Surgical Management}

Surgical therapies are typically reserved for corneal infections unresponsive to medical therapy, or in cases of impending or confirmed perforation. In a retrospective review from Iowa, $44 \%$ of individuals with FK had persistent disease that required TKP, and of these $47 \%$ had a failed graft at 15 months. Smaller graft sizes were more likely to remain clear [54]. Further studying the effects of graft size, another group found a higher incidence of epithelial, stromal, or endothelial rejection and secondary glaucoma when the grafts were larger than $8 \mathrm{~mm}$ [55], consistent with previous studies.

Immunosuppression may decrease the risk of rejection after TPK, but corticosteroids are avoided by many surgeons due to possible persistent fungal elements and the risk of recurrent infection. A recent study revealed a vital role of calcineurin in the virulence of Candida sp. [56] This makes the calcineurin inhibitor and immunomodulator Tacrolimus potentially effective at providing some antifungal activity while delivering the desired immunosuppression. This also supports the rationale for utilizing topical cyclosporine, another calcineurin inhibitor, as prophylaxis of transplant rejection in cases of therapeutic keratoplasty.

Also of concern to corneal surgeons, donor tissue can be contaminated with fungi. From 2007 to 2010, there were 31 reported cases of post-operative FK out of 221,664 transplants. The only culture-positive fungal organisms were Candida sp. Donor characteristics demonstrated that $32 \%$ were diabetic, $35 \%$ were hospitalized prior to death, and $29 \%$ were on respirator support. A notable data point was that out of the 31 cases, 22 of the fellow donor eyes were cultured; 16 were contaminated with the same species of Candida. 15 of those corneas were transplanted; 10 resulted in FK or endophthalmitis. This highlights the utility of performing fungal cultures on donor tissue, and also the need for rapid reporting of adverse events to the eye bank.

Because of the low and relatively stable incidence, the authors recommended continued monitoring without adding supplemental anti-fungal agents to corneal storage media $[57 \bullet \bullet]$. Future studies would be required to confirm the safety and efficacy of any proposed antifungal prophylaxis for donor tissue.

As an alternative to penetrating keratoplasty, 10 patients were treated with debriding keratectomy with autologous conjunctival graft; the subjects' visual acuities at 3 months all improved from their nadir. The surgical management was guided by evaluation of corneas by anterior segment OCT displaying ulcer depths that were $1 / 3-1 / 2$ total corneal thickness, infiltrate extending no more than $80 \mathrm{um}$ further, and basal diameter of 3-6 $\mathrm{mm}$ [58].

Another question that has been addressed in recent literature pertains to timing of surgical intervention. A case series from Chang Gung Memorial compared medical treatment versus early (within 1 week) superficial keratectomy of maximum depth of $1 / 2$ to $2 / 3$ of the total corneal thickness. The authors reported comparable baseline severity of keratitis in both groups, but did note a statistically significant worse VA in the patients who received medical treatment only. In the early keratectomy group, they found a decrease in hospital stay by a mean of 20.5 days, a decrease in disease duration by a mean of 25.5 days, a decrease in the hospital cost from decreased ward and adjuvant procedural fees by median 29,884.1 new Taiwanese dollars (986.18 USD by current conversion), and improved VA in early superficial keratectomy versus medical management (all $p<0.001$ ). It should also be noted that in their keratectomy group there was never a 
need for amniotic membrane or patch graft, nor did they have problems with subsequent glaucoma or recurrence [59].

\section{Experimental}

Due to the challenging nature of this form of keratitis, and less than ideal outcomes with current treatment in many cases, various experimental therapies are being investigated.

Corneal collagen cross-linking (CXL) in conjunction with photo-activated riboflavin (PAR) biomechanically stabilizes the cornea in the setting of ectastia from keratoconus or as a complication of post-operative refractive laser surgery [60]. As enzymatic degradation of corneal integrity occurs in infectious keratitis, CXL with PAR has been investigated in refractory cases (bacterial, acnathamoebal, as well as fungal) [61-63]. In an in vitro study on CXL/PAR treatment of $C$. albicans and $F$. solani, phenotypic changes were noted on growth plates for both fungal agents, but no difference was noted by cell counter analysis of morphology between untreated fungal elements and those treated [64•]. While the preliminary human studies involve small numbers, the efficacy for amoebic and FKs does not appear to be dramatic. Notably, caution is also needed as the UV exposure may lead to reactivation of latent herpes virus infection [65].

Investigations into the use of nanoparticle technology with terbinafine and silver have shown promise [66, 67]. There has been an attempt to develop an antimicrobial contact lens incorporating melimine into the lens substance [68]. Lactoferricin B and short imidazolium chains have shown anti-biofilm properties and have been studied as a possible component to add to contact lens cleaning solution or as an adjunct to help increase the susceptibility of fungi to anti-fungal agents $[69,70]$. Even topical aqueous garlic extract has been used in in vivo rabbit models infected with A. flavus [71].

\section{Conclusion}

Fungal Keratitis has proven to be a continually challenging ocular disease for patients, providers, and society. The most common etiologic agents have been identified, but making the diagnosis clinically has proven to be inconsistent. Once a fungal etiology is suspected; traditional techniques of identification may not be sensitive or rapid enough for optimal outcomes. Recent advances in techniques such as in vivo confocal microscopy and the evolution of PCR promise to increase the speed and accuracy of diagnosis. A common weakness of microscopic stains and even confocal microscopy is the subjective nature of the interpretation of the results, which may explain some of the variability of results between various authors. Within this context, molecular techniques that are more objective and reproducible may avoid the variation inherent in interpretation of the other diagnostic modalities. The remaining obstacles include standardization of methodologies, meeting regulatory requirements, and access at a cost that is appropriate for the benefit provided.

A greater understanding of the pathophysiologic mechanisms highlight new areas where therapeutics may be directed from both the host and fungal standpoints. Understanding the epidemiology as well as the pathophysiology of contact-lens-associated FK is an example of this merging of disparate knowledge pools, resulting in a concrete change in policy and a tangible impact on disease burden.

Landmark studies such as MUTT have published data that are contradictory to previous expert opinions regarding topical medical treatment, and the treatment continues to evolve. Topical Natamycin may still be the empiric agent of choice for filamentous organisms, but better predictors of antibiotic sensitivity, newer antifungal agents, and novel drug delivery strategies will likely continue to decrease the likelihood of medical treatment failure.

Disclosure Donald Stone and Jeremy F. Tan have reported no conflicts of interest. This work was supported in part by an unrestricted grant from Research to Prevent Blindness to the University of Oklahoma Dean McGee Eye Institute.

Human and Animal Rights and Informed Consent This article does not contain any studies with human or animal subjects performed by any of the authors.

\section{References}

Papers of particular interest, published recently, have been highlighted as:

- Of importance

•- Of major importance

1. Dalmon $\mathrm{C}$, et al. The clinical differentiation of bacterial and fungal keratitis: a photographic survey. Invest Ophthalmol Vis Sci. 2012;53(4):1787-91.

2. Kampitak K, Patrasuwan S, Kongsomboon K. Cost evaluation of corneal ulcer treatment. J Med Assoc Thai. 2013;96(4):456-9.

3. Prajna VN, et al. Economic analysis of corneal ulcers in South India. Cornea. 2007;26(2):119-22.

4. Keay L, et al. Microbial keratitis predisposing factors and morbidity. Ophthalmology. 2006;113(1):109-16.

5. Karsten E, Watson SL, Foster LJR. Diversity of microbial species implicated in keratitis: a review. Open Ophthalmol J. 2012;6:110.

6. Tasanee $S$, et al. Predisposing factors and etiologic diagnosis of ulcerative keratitis. Cornea. 2008;27(3):283-7.

7. Lu XH, et al. Aetiology analyses of 334 cases fungal keratitis. Chin J Ophthalmol (Zhonghua yan ke za zhi). 2013;49(1):12-5. 
8. Srinivasan M, et al. Epidemiology and aetiological diagnosis of corneal ulceration in Madurai, South India. Br J Ophthalmol. 1997;81(11):965-71.

9. Ansari Z, Miller D, Galor A. Current thoughts in fungal keratitis: diagnosis and treatment. Curr Fungal Infect Rep. 2013;7(3):209-18.

10. Garg P. Microsporidia infection of the cornea: a unique and challenging disease. Cornea. 2013;32(Suppl 1):S33-8.

11. - Das $\mathrm{S}$, et al. Diagnosis, clinical features and treatment outcome of microsporidial keratoconjunctivitis. Br J Ophthalmol. 2012;96(6): 793-5. This article highlights an uncommon, but likely underdiagnosed, cause of infection keratoconjunctivitis, microsporidia. Since routine cultures do not detect these organisms, an increase index of suspicion is required.

12. Font LR, et al. Corneal microsporidiosis. Report of case, including electron microscopic observations. Ophthalmology. 2000;107(9): 1769-75.

13. Leal SM, Pearlman E. The role of cytokines and pathogen recognition molecules in fungal keratitis: insights from human disease and animal models. Cytokine. 2012;58(1):107-11.

14. Wang N, et al. Association of TLR2 and TLR4 gene single nucleotide polymorphisms with fungal keratitis in Chinese Han population. Curr Eye Res. 2014;39(1):47-52.

15. Gower EW, et al. Trends in fungal keratitis in the United States, 2001 to 2007. Ophthalmology. 2010;117(12):2263-7.

16. Bullock JD, et al. Effects of time, temperature, and storage container on the growth of Fusarium species: implications for the worldwide fusarium keratitis epidemic of 2004-2006. Arch Ophthalmol. 2011;129(2):133-6.

17. • Bullock JD, et al. Mechanism of drug failure in fusarium keratitis, 2004-2006. N Engl J Med. 2014;370(1):88-9. This important article highlights the need for critical review of every aspect of clinical care, from device design and testing, implementation, and patient compliance to post-marketing surveillance.

18. Clavet CR, et al. Impact of contact lens materials on multipurpose contact lens solution disinfection activity against Fusarium solani. Eye Contact Lens. 2012;38(6):379-84.

19. Kuzman T, et al. Lens wearers non-compliance; is there an association with lens case contamination? Cont Lens Anterior Eye. 2014;37(2):99-105.

20. - Zhang X, et al. Keratitis-associated fungi form biofilms with reduced antifungal drug susceptibility. Invest Ophthalmol Vis Sci. 2012;53(12):7774-8. Biofilm formation is one of the primary mechanisms through which fungi evade the immune response and establish infection. This is important for understanding the pathogenesis of infections, and may represent therapeutic targets in the future.

21. Wang FY. Staining with methylthioninium chloride for the diagnosis of fungal keratitis. Exp Ther Med. 2013;6(5):1229-32.

22. Reddy KA, et al. Is blood agar an alternative to sabouraud dextrose agar for the isolation of fungi in patients with mycotic keratitis. Int Ophthalmol. 2012;33(3):251-4.

23. Takezawa Y, et al. Effectiveness of in vivo confocal microscopy in detecting filamentous fungi during clinical course of fungal keratitis. Cornea. 2010;29(12):1346-52.

24. Villani E, et al. In vivo confocal microscopy of the ocular surface: from bench to bedside. Curr Eye Res. 2014;39(3):213-31.

25. Tananuvat $\mathrm{N}$, et al. Prospective comparison between conventional microbial work-up vs PCR in the diagnosis of fungal keratitis. Eye. 2012;26(10):1337-43.

26. Eleinen KGA, et al. Polymerase chain reaction-guided diagnosis of infective keratitis: a hospital based study. Curr Eye Res. 2012;37(11):1005-11.

27. Jordanides NE, et al. A prospective study of real-time panfungal PCR for the early diagnosis of invasive fungal infection in haematooncology patients. Bone Marrow Transpl. 2005;35(4):389-95.
28. Basková $\mathrm{L}$, et al. The pan-AC assay: a single-reaction real-time PCR test for quantitative detection of a broad range of Aspergillus and Candida species. J Med Microbiol. 2007;56(Pt 9):1167-73.

29. Rakeman JL, et al. Multilocus DNA sequence comparisons rapidly identify pathogenic molds. J Clin Microbiol. 2005;43(7): 3324-33.

30. Schabereiter-Gurtner C, et al. Development of novel real-time PCR assays for detection and differentiation of eleven medically important Aspergillus and Candida species in clinical specimens. J Clin Microbiol. 2007;45(3):906-14.

31. Goldschmidt P, et al. New strategy for rapid diagnosis and characterization of fungal infections: the example of corneal scrapings. PLoS One. 2012;7(7):e37660.

32. Hsiao CR, et al. Identification of medically important molds by an oligonucleotide array. J Clin Microbiol. 2005;43(8):3760-8.

33. Kuo MT, et al. A highly sensitive method for molecular diagnosis of fungal keratitis. Ophthalmology. 2012;119(12):2434-42.

34. Krishnan T, et al. Gender differences in re-epithelialisation time in fungal corneal ulcers. Br J Ophthalmol. 2012;96(1):137-8.

35. Jetton JA, et al. Effects of tobacco smoking on human corneal wound healing. Cornea. 2014;33(5):453-6.

36. Prajna NV, et al. Predictors of outcome in fungal keratitis. Eye. 2012;26(9):1226-31.

37. Prajna NV, et al. Differences in clinical outcomes in keratitis due to fungus and bacteria. JAMA Ophthalmol. 2013;131(8):1088.

38. Kurbanyan $\mathrm{K}$, et al. Corneal nerve alterations in acute acanthamoeba and fungal keratitis: an in vivo confocal microscopy study. Eye. 2011;26(1):126-32.

39. Henry CR, et al. Infectious keratitis progressing to endophthalmitis. Ophthalmology. 2012;119(12):2443-9.

40. Ramakrishnan T, et al. Factors affecting treatment outcomes with voriconazole in cases with fungal keratitis. Cornea. 2013; 32(4):445-9.

41. Parchand S, et al. Voriconazole for fungal corneal ulcers. Ophthalmology. 2012;119(5):1083.

42. • Prajna NV, et al. The mycotic ulcer treatment trial: a randomized trial comparing natamycin vs voriconazole mycotic ulcer treatment trial. JAMA Ophthalmol. 2013;131(4):422-9. This prospective trial overcame many of the weaknesses in previous retrospective studies, and an unexpected finding was the superiority of natamycin over voriconazole for infections attributed to Fusarium. The generalizability of these findings may depend on local susceptibility patterns, but are an important guide to therapy.

43. Sun CQ, et al. Expert prior elicitation and Bayesian analysis of the mycotic ulcer treatment trial I. Invest Ophthalmol Vis Sci. 2013;54(6):4167-73.

44. - Lalitha P, et al. Organism, minimum inhibitory concentration, and outcome in a fungal corneal ulcer clinical trial. Cornea. 2012;31(6):662-7. Antifungal sensitivity testing is not commonly performed in clinical scenarios, but the Minimum Inhibitory Concentration was predictive of corneal perforation in this substudy of the MUTT trial.

45. Oechsler R, et al. Fusarium keratitis in Brazil: genotyping, in vitro susceptibilities, and clinical outcomes. Clin Ophthalmol. 2013;7:1693.

46. Abdul Rasool BK, Salmo HM. Development and clinical evaluation of clotrimazole- $\beta$-cyclodextrin eyedrops for the treatment of fungal keratitis. AAPS PharmSciTech. 2012;13(3):883-9.

47. Habib FS, et al. Liposomes as an ocular delivery system of fluconazole: in-vitro studies. Acta Ophthalmol. 2010;88(8): 901-4.

48. Abdel-Rhaman MS, et al. A new long-acting liposomal topical antifungal formula: human clinical study. Cornea. 2012;31(2): $126-9$. 
49. Qu LH, Li LM, Xie HP. Toxicity and pharmacokinetics of intrastromal injection of amphotericin B in a rabbit model. Curr Eye Res. 2013;39(4):340-7.

50. Prakash G, et al. Evaluation of intrastromal injection of voriconazole as a therapeutic adjunctive for the management of deep recalcitrant fungal keratitis. Am J Ophthalmol. 2008;146(1):56-9.

51. Sharma N, et al. Comparative evaluation of topical versus intrastromal voriconazole as an adjunct to natamycin in recalcitrant fungal keratitis. Ophthalmology. 2013;120(4):677-81.

52. Shen YC, et al. Pharmacokinetics of intracameral voriconazole injection. Antimicrob Agents Chemother. 2009;53(5):2156-7.

53. Mittal V, Mittal R. Intracameral and topical voriconazole for fungal corneal endoexudates. Cornea. 2012;31(4):366-70.

54. Rogers GM, et al. Outcomes of treatment of fungal keratitis at the university of Iowa hospitals and clinics: a 10-year retrospective analysis. Cornea. 2013;32(8):1131-6.

55. Li C, et al. Effect of corneal graft diameter on therapeutic penetrating keratoplasty for fungal keratitis. Int J Ophthalmol. 2012;5(6):698.

56. Zhang J, et al. Calcineurin is required for pseudohyphal growth virulence, and drug resistance in Candida Lusitaniae. PLoS One. 2012;7(8):e44192.

57. - Aldave AJ, et al. Report of the eye bank association of America medical advisory board subcommittee on fungal infection after corneal transplantation. Cornea. 2013;32(2):149-54. The systems-based approach to preventing fungal infection after keratoplasty is the topic of ongoing surveillance and debate; this report found a small but statistically insignificant increase in infections related to fungal contamination of donor tissue, and deferred consideration of adding antifungal agents to tissue storage media until there is better data regarding necessity and safety of this broad change in eye bank protocol.

58. Sun GH, et al. Clinical observation of removal of the necrotic corneal tissue combined with conjunctival flap covering surgery under the guidance of the AS-OCT in treatment of fungal keratitis. Int J Ophthalmol. 2012;5(1):88.

59. Lin HC, et al. Early keratectomy in the treatment of moderate fusarium keratitis. PLoS One. 2012;7(8):e42126.

60. Nguyen MK, Chuck RS. Corneal collagen cross-linking in the stabilization of PRK, LASIK, thermal keratoplasty, and orthokeratology. Curr Opin Ophthalmol. 2013;24(4):291-5.
61. Li ZW, et al. Riboflavin/ultraviolet light-mediated crosslinking for fungal keratitis. Br J Ophthalmol. 2013;97(5):669-71.

62. Müller L, et al. Corneal cross-linking as supplementary treatment option in melting keratitis: a case series. Klinische Monatsblätter Für Augenheilkunde. 2012;229(4):411-5.

63. Panda A, Krishna SN, Kumar S. Photo-activated riboflavin therapy of refractory corneal ulcers. Cornea. 2012;31(10):1210-3.

64. - Kashiwabuchi RT, et al. Assessment of fungal viability after long-wave ultraviolet light irradiation combined with riboflavin administration. Graefe's Arch Clin Exp Ophthalmol. 2012;251(2):521-7. This experimental model supports the clinical observation that corneal collagen crosslinking via Photoactivated riboflavin may not be beneficial in fungal keratitis, or may require modifications of current treatment protocols to improve efficacy.

65. Price MO, et al. Photoactivated riboflavin treatment of infectious keratitis using collagen cross-linking technology. J Refract Surg. 2012;28(10):706-13.

66. Tayel SA, et al. Positively charged polymeric nanoparticle reservoirs of terbinafine hydrochloride: preclinical implications for controlled drug delivery in the aqueous humor of rabbits. AAPS PharmSciTech. 2013;14(2):782-93.

67. $\mathrm{Xu} \mathrm{Y}$, et al. In vitro antifungal activity of silver nanoparticles against ocular pathogenic filamentous fungi. J Ocul Pharmacol Ther. 2013;29(2):270-4.

68. Dutta D, et al. Broad spectrum antimicrobial activity of melimine covalently bound to contact lenses. Invest Ophthalmol Vis Sci. 2013;54(1):175-82.

69. Sengupta J, et al. Effects of Lactoferricin B against keratitis-associated fungal biofilms. J Infect Chemother. 2012;18(5):698-703.

70. Liu LH, et al. Short imidazolium chains effectively clear fungal biofilm in keratitis treatment. Biomaterials. 2013;34(4):1018-23.

71. Ismaiel AA, et al. Efficacy of aqueous garlic extract on growth, aflatoxin b1 production, and cyto-morphological aberrations of Aspergillus flavus, causing human ophthalmic infection: topical treatment of A. flavus keratitis. Braz J Microbiol. 2012;43(4): 1355-64.

72. Bhadange $\mathrm{Y}$, et al. Role of liquid culture media in the laboratory diagnosis of microbial keratitis. Am J Ophthalmol. 2013;156(4): $745-51$. 